\title{
Producción de etanol a partir de piñas de rechazo de cultivos del Chocó
}

\author{
Juan Fernando Murcia P ${ }^{1}$; Alba N. Ardila A²; Rolando Barrera-Zapata ${ }^{1 *}$ \\ ${ }^{1}$ Grupo CERES Agroindustria e Ingeniería, Facultad de Ingeniería, Universidad de Antioquia UdeA, \\ calle 70 n. ${ }^{\circ}$ 52-21, Medellín, Colombia. \\ ${ }^{2}$ Grupo CAMER, Facultad de Ciencias Básicas Sociales y Humanas, Politécnico Colombiano Jaime Isaza Cadavid. \\ Apartado Aéreo 49-32, Medellín, Colombia \\ *rolando.barrera@udea.edu.co
}

Fecha recepción: diciembre 2 de 2019

Fecha aceptación: enero 24 de 2020

\begin{abstract}
Resumen
En este trabajo se estudió la producción de etanol a partir de jugo de piñas de rechazo (frutos que no cumplen las normas técnicas para su comercialización) del departamento del Chocó. La materia prima se caracterizó y se usó en fermentaciones donde se evaluó el efecto del uso de levadura comercial, la dilución del medio y la adición de suplementos sobre la cantidad de alcohol producido a las $72 \mathrm{~h}$ de reacción. El perfil de azúcares y la caracterización bromatológica de esta variedad de piña sugiere que corresponde a un sustrato adecuado para la obtención de alcohol sin necesidad de nutrientes o suplementos adicionales. La producción de etanol utilizando $S$. cerevisiae fue cercana a los $65 \mathrm{gL}^{-1} \mathrm{en}_{\text {un }}$ medio de jugo de piña sin diluir, sin adición de nutrientes y sin control de temperatura (entre 23 y $38^{\circ} \mathrm{C}$ ) ni $\mathrm{pH}$ (entre 4,0 y 4,5$)$ durante la reacción. Bajo condiciones de temperatura $\left(30^{\circ} \mathrm{C}\right)$ y $\mathrm{pH}(4,5)$ controlados, el tiempo requerido para alcanzar los mismos rendimientos disminuyó considerablemente (menos de 15 horas). Se espera que estos resultados permitan proponer y desarrollar alternativas para la valorización de los residuos del cultivo de piña de la región y para la disminución del impacto ambiental negativo que representan.
\end{abstract}

Palabras clave: Bioetanol; Residuos de Piña; Saccharomyces Cerevisiae; Valoración de Desecho. 


\title{
Ethanol production from pineapple rejection of Choco crops
}

\begin{abstract}
In this work it was studied the production of ethanol from rejected-pineapple juice (that is, fruits that do not meet the technical standards for marketing) from the department of Chocó. The raw material was characterized and used in fermentations where the effect of the use of commercial yeast, the dilution of the medium and the addition of supplements on the amount of alcohol produced at $72 \mathrm{~h}$ of reaction was evaluated. The sugar profile and the characterization of this pineapple variety suggests that it corresponds to a suitable substrate for obtaining alcohol without the need for additional nutrients or supplements. Ethanol production using $S$. cerevisiae was close to $65 \mathrm{gL}^{-1}$ in a medium of undiluted pineapple juice, without the addition of nutrients and without temperature (between 23 and $38^{\circ} \mathrm{C}$ ) or pH (between 4.0 and 4.5) control. Under controlled temperature $\left(30^{\circ} \mathrm{C}\right)$ and $\mathrm{pH}(4.5)$ conditions, the time required to reach the same yields decreased considerably (less than 15 hours). These results are expected to be useful in the propose and develop of alternatives for the recovery of pineapple crop residues in the region and to reduce the negative environmental impact that they represent.
\end{abstract}

Keywords: Bioethanol; Pineapple Residues; Saccharomyces Cerevisiae; Waste Valorization.

\section{Produção de etanol a partir da rejeição de abacaxi de Choco}

\begin{abstract}
Resumo
Neste trabalho estudamos a produção de etanol a partir de suco de abacaxi do departamento de Chocó (ou seja, frutas que não atendem aos padrões técnicos de marketing). A matéria-prima foi caracterizada e utilizada em fermentações onde foram avaliados o efeito do uso de levedura comercial, a diluição do meio e a adição de suplementos na quantidade de álcool produzido às $72 \mathrm{~h}$ de reação. $\mathrm{O}$ perfil de açúcar e a caracterização bromatológica dessa variedade de abacaxi sugerem que ela corresponde a um substrato adequado para a obtenção de álcool sem a necessidade de nutrientes ou suplementos adicionais. A produção de etanol com $S$. cerevisiae foi próxima a $65 \mathrm{gL}^{-1} \mathrm{em}$ meio de suco de abacaxi não diluído, sem adição de nutrientes e sem controle de temperatura $\left(23-38{ }^{\circ} \mathrm{C}\right)$ ou $\mathrm{pH}(4,0-4,5)$. Sob condições de temperatura controlada $\left(30^{\circ} \mathrm{C}\right)$ e $\mathrm{pH}(4,5)$, o tempo necessário para atingir os mesmos rendimentos diminuiu consideravelmente (menos de 15 horas). Esses resultados devem propor e desenvolver alternativas para a recuperação de resíduos de abacaxi na região e reduzir o impacto ambiental negativo que eles representam.
\end{abstract}

Palavras-chave: Bioetanol; Resíduos de Abacaxi; Saccharomyces Cerevisiae; Avaliação de Resíduos. 


\section{Introducción}

En Colombia se tienen actualmente destinadas alrededor de 32.000 hectáreas para el cultivo de piña, con una producción que supera el millón de toneladas anuales [1,2]. Casi el $60 \%$ de esa producción se destina para su consumo en fresco, mientras que el resto se exporta o se transforma para obtener productos derivados (como mermeladas, concentrados o enlatados) que se comercializan principalmente en mercados locales [1]. Cuando la piña se consume en fresco o se procesa para obtener derivados, aproximadamente el $50 \%$ en peso de la fruta se descarta como residuo (pulpa residual, corona, cáscara y corazón) [3]. Adicionalmente, diferentes factores, como la topografía, el clima y el estado de desarrollo, en cuanto a infraestructura y vías de acceso de algunas regiones del país donde se da la producción de piña (como, por ejemplo, el Chocó, el Urabá antioqueño y el Casanare, entre otras), generan un alto desperdicio de la fruta cosechada, denominado piña de rechazo, y que consiste en las frutas que no logran los estándares o características mínimas para su comercialización - las que no alcanzan a comercializarse antes de iniciar su deterioro natural. En algunas de estas regiones se ha reportado que la piña de rechazo puede llegar a representar casi hasta el $50 \%$ de la cosecha [4]. Así mismo, dadas las condiciones de desarrollo propias de esas regiones, los desechos asociados al cultivo y comercialización de la piña suelen terminar en botaderos a cielo abierto o en fuentes hídricas, lo cual genera un problema ambiental por las demandas químicas y biológicas de oxígeno de este tipo de residuos $[3,4]$.

En literatura reciente se encuentran diversos estudios que demuestran el potencial de recuperación o de uso de los residuos del cultivo y comercialización de piña para la producción o generación de múltiples productos con diferente valor agregado [5-8]; así, se destaca, por ejemplo,

la posibilidad de obtener bromelina, pectina, goma de xantano, vainillina, xilosa, xilitol, celulosa, glucosa, carbón activado, fibras, ácidos orgánicos, alcoholes, suplementos animales, entre muchos otros, bien sea a través de procesos físicos, químicos o biotecnológicos. Dentro de la variedad de productos que potencialmente pueden obtenerse a partir de residuos agroindustriales, como los derivados del cultivo y procesamiento de la piña, el etanol recibe especial atención, debido a su uso e importancia en industrias de bebidas fermentadas, industrias alimenticias e industrias farmacéuticas, entre otras
[9-10]. Adicionalmente, entre los principales usos del etanol, se destaca su uso como aditivo para gasolina o como combustible puro; de hecho, actualmente es el biocombustible de mayor producción a nivel global. Como dato estadístico, la producción de bioetanol en 2018 alcanzó los 60.000 millones de litros en Estados Unidos, mientras que en Colombia la producción estuvo cerca a los 500 millones de litros [11].

La fermentación de residuos agroindustriales para la obtención de alcoholes puede darse a través de diversas clases de microorganismos que incluyen levaduras (por ejemplo, Saccharomyces cerevisiae, Saccharomyces ellipsodides, Saccharomyces carlsbegensis, Saccharomyces fragilis, Saccharomyces ovarum, Saccharomyces pastorianus, Schizosaccharomyces pombe, Kluyveromyces marxianus IMB3 y Pichia stipitis), bacterias (como Escherichia coli, Zymomonas mobilis, Thermoanaerobacter mathrani, Clostridium acetobutylicum y Clostridium beijerickil) y hongos - mohos (como Aspergillus oryzae, Aspergillus awamori y Candida shehatae) [12]. Las levaduras suelen ser los microorganismos más utilizados dada su relativa alta resistencia a las condiciones típicas de los medios de reacción requeridos, cuyas temperaturas pueden rondar o superar los $35^{\circ} \mathrm{C}$ y donde la concentración de alcohol puede llegar hasta el $18 \%$ v. Además, estas levaduras se consideran aditivos alimenticios seguros para consumo humano, por lo que resultan adecuadas para producir bebidas alcohólicas [13].

Específicamente, para la obtención de etanol utilizando como materia prima residuos del cultivo de piña, se ha reportado el uso de microorganismos como Saccharomyces cerevisiae y Zymomonas mobilis. Ambos microorganismos pueden llegar a producir alrededor del $8 \%$ v de etanol en periodos que oscilan entre las 20 y las 48 h $[9,10,14]$. Adicionalmente, con la cepa de Saccharomyces cerevisiae se han realizado estudios para la producción de etanol en sistemas continuos utilizando como materia prima jugo prensado de residuos generados en la producción de piña enlatada [15]. Los rendimientos a etanol reportados equivalen a producciones cercanas a los $59,0 \mathrm{gL}^{-1}$ sin necesidad de adicionar suplementos al medio de cultivo ni regular el $\mathrm{pH}$. No obstante, dichos rendimientos pueden variar de acuerdo con la especie o variedad de piña que se utilice como materia prima. Para el caso de las piñas de rechazo del departamento del Chocó, no se encontró en la literatura ningún reporte relacionado con su 
caracterización ni aprovechamiento en la producción de alcohol u otros productos de valor agregado.

En este trabajo se evalúa la producción de etanol a partir de jugo de piñas de rechazo del departamento del Chocó, con el fin de proponer alternativas que permitan la valorización de este tipo de residuos y la disminución del impacto ambiental negativo que representan. Inicialmente, se presenta la caracterización bromatológica de esta variedad de piñas y, a continuación, la evaluación de parámetros como el uso de levadura comercial, la concentración del jugo de piña utilizado en las fermentaciones y el efecto de la adición de nutrientes al medio de reacción. Finalmente, se obtiene un perfil experimental de la formación de alcohol y la desaparición de azúcares en el tiempo bajo las mejores condiciones encontradas para la fermentación del jugo de piña de rechazo.

\section{Experimental}

\section{Obtención, preparación y caracterización de ma- teriales}

La materia prima utilizada consistió en piñas de rechazo (frutos que no cumplen la NTC 729-1) provenientes de las plantaciones de Tutunendo, corregimiento de Quibdó en el departamento del Chocó. Se les retiró el pedúnculo y la corona a las piñas. Las partes restantes (pulpa, corazón y cáscara) se utilizaron para la producción del jugo en un extractor de jugos casero (Oster, modelo 3168). Una vez obtenido el jugo, se pasó por un filtro con tamaño de poro de $0,5 \mathrm{~mm}$ y se ajustó su $\mathrm{pH}$ a 4,5 utilizando solución de hidróxido de sodio $1 \mathrm{M}$; después, se esterilizó en un autoclave a 15 psi por $20 \mathrm{~min}, \mathrm{y}$, posteriormente, se almacenó a una temperatura aproximada a $6{ }^{\circ} \mathrm{C}$ hasta su uso. El material retenido en el filtro se caracterizó determinando su porcentaje de humedad por el método de peso seco, según AOAC 20.013; el pH (por potenciometría), según el método A.O.A.C 981.12, el porcentaje de grasa por el método Soxhlet según AOAC 39.1.05; el porcentaje de fibra cruda por hidrólisis ácida, alcalina y calcinación según A.O.A.C 7.073; el porcentaje de proteína por el método Kjeldahl, según AOAC 2.057, el porcentaje de cenizas por calcinación según AOAC 31.012; el porcentaje de carbohidratos por diferencia y los sólidos totales por el método AOAC 932.12. Por su parte, sobre el jugo se realizó la cuantificación de azúcares (sacarosa, glucosa y fructosa). La cuantificación de azúcares y etanol se realizó por HPLC (Shimadzu) en una columna Waters IC-Pack ion exclusión y un detector de índice de refracción Shimadzu, modelo RID-10 A. Se empleó como fase móvil ácido sulfúrico $0,005 \mathrm{~N}$ con un flujo de bombeo de $0,6 \mathrm{mLmin}^{-1}$.

\section{Preparación del inóculo}

Para realizar la fermentación de la piña, se utilizó una levadura comercial liofilizada (Saccharomyces cerevisiae). El inóculo correspondióal 10\% del medio. Se preparó un jugo de piña sin diluir, incubándolo por $24 \mathrm{~h}$ con una velocidad de agitación de $150 \mathrm{rpm}$ y subcultivándolo cada 8 días a temperatura ambiente en cajas de Petri con agar Sabouroud. Para efectos comparativos, también se realizaron fermentaciones utilizando inóculos preparados de manera similar, pero con porciones aisladas de la misma variedad de piña en lugar de la levadura, y, de esta manera, aprovechar los microorganismos de tipo nativo que la fruta posee y que son responsables de su fermentación natural.

\section{Medios de cultivo}

Se evaluaron tres medios de cultivo con diferente concentración de jugo de piña de rechazo para la producción de etanol de la siguiente manera: $50 \% \mathrm{v}$ (50\% jugo de piña, $50 \%$ agua destilada), $75 \%$ v (75\% jugo de piña, $25 \%$ agua destilada) y $100 \%$ (jugo sin diluir). Cada uno de estos medios se evaluó con y sin adición de nutrientes. Para la adición de nutrientes se siguieron las recomendaciones de Riviera et al. [16], es decir, se utilizaron $5,0 \mathrm{gL}^{-1} \mathrm{de}$ extracto de levadura; $5,0 \mathrm{gL}^{-1}$ de $\mathrm{K}_{2} \mathrm{HPO}_{4}, 1,5 \mathrm{gL}^{-1} \mathrm{de}$ $\mathrm{NH}_{4} \mathrm{Cl}, 0,5 \mathrm{gL}^{-1}$ de $\mathrm{MgSO}_{4} \times 7 \mathrm{H}_{2} \mathrm{O}$ y $1,2 \mathrm{gL}^{-1}$ de $\mathrm{KCl}$.

\section{Fermentaciones}

Para las fermentaciones se utilizaron Erlenmeyers de $250 \mathrm{~mL}$. Se utilizó en cada caso $100 \mathrm{~mL}$ del respectivo medio de cultivo a temperatura ambiente, sin control de temperatura ni del $\mathrm{pH}$ durante el proceso. Al inicio de la reacción se registró la temperatura en $23^{\circ} \mathrm{C}$ y el pH en 4,5. No se registraron los cambios de estas variables durante el proceso, pero al final del experimento la temperatura registró $38{ }^{\circ} \mathrm{C}$, y el pH estuvo cerca a 4,0. La agitación se mantuvo constante en $150 \mathrm{rpm}$ mediante un agitador orbital durante $72 \mathrm{~h}$. Los experimentos realizados se resumen en la tabla 1 . Se realizaron dos réplicas para cada uno de los experimentos 1 al 6 , y una réplica para cada uno de los experimentos 7 al 12 . Con el promedio de las desviaciones estándar de los experimentos similares, se estimó un margen de error para la cuantificación del alcohol producido. 
rev. ion. 2020;33(1):47-56. Bucaramanga (Colombia).

Tabla 1. Experimentos para evaluar la producción de etanol a partir de jugo de piña de rechazo.

\begin{tabular}{ccccc}
\hline Experimento & Microorganismo & Medio de cultivo & Adición nutrientes & Réplicas \\
\hline 1 & S. cerevisiae & $50 \%(\mathrm{vol})$ & No & 2 \\
2 & S. cerevisiae & $50 \%(\mathrm{vol})$ & Sí & 2 \\
3 & S. cerevisiae & $75 \%(\mathrm{vol})$ & No & 2 \\
4 & S. cerevisiae & $75 \%(\mathrm{vol})$ & Sí & 2 \\
5 & S. cerevisiae & $100 \%$ & No & 2 \\
6 & S. cerevisiae & $100 \%$ & Sí & 2 \\
7 & Nativo & $50 \%(\mathrm{vol})$ & No & 1 \\
8 & Nativo & $50 \%(\mathrm{vol})$ & Sí & 1 \\
9 & Nativo & $75 \%(\mathrm{vol})$ & No & 1 \\
10 & Nativo & $75 \%(\mathrm{vol})$ & Sí & 1 \\
11 & Nativo & $100 \%$ & No & 1 \\
12 & Nativo & $100 \%$ & Sí & 1 \\
\hline
\end{tabular}

El experimento que arrojó los mejores resultados (mayor producción de etanol) se reprodujo en un biorreactor controlado (New BrunsWick, BIOFLO 110), donde se mantuvo la temperatura en $30^{\circ} \mathrm{C}$ y el $\mathrm{pH}$ en 4,5 durante todo el tiempo de la fermentación. En este se tomó una muestra inmediatamente después de la inoculación (tiempo cero) y luego se procedió con muestreos cada $2 \mathrm{~h}$ para registrar los perfiles de la producción de etanol y consumo de sustratos (azúcares).

\section{Resultados}

El jugo obtenido a partir de las piñas de rechazo del Chocó presentó pH de 3,9, densidad de $1,11 \mathrm{gcm}^{-1}$ y $21,7^{\circ} \mathrm{Bx}$. Los grados Brix resultan considerablemente superiores a los reportados para otras variedades de piña que suelen estar entre 13 y $17^{\circ} \mathrm{Bx}$ [17]. El rendimiento promedio en la obtención del jugo fue de $76 \mathrm{~L} / 100 \mathrm{~kg}$, valor superior al esperado (aprox. $50 \mathrm{~L} / 100 \mathrm{~kg}$ ) según [18]. El análisis bromatológico de la materia prima (\%másico, en húmedo y con cenizas) se presenta en la tabla 2.

Tabla 2. Análisis bromatológico de las piñas de rechazo utilizadas para la obtención de etanol.

\begin{tabular}{cc}
\hline Componente & \%Másico \\
\hline Humedad & 82,5 \\
Grasa & 0,3 \\
Fibra cruda & 0,7 \\
Proteína & 0,4 \\
Cenizas & 0,4 \\
Carbohidratos & 15,7 \\
\hline
\end{tabular}

Por su parte, el perfil de azúcares de la materia prima (piñas de rechazo) se muestra en la tabla 3. Al comparar la cantidad de glucosa y fructosa del jugo de piña de rechazo con la cantidad presente en las piñas de buena calidad comercializadas $\left(120,9 \mathrm{gL}^{-1}\right.$ para la glucosa y $90,45 \mathrm{gL}^{-1}$ para la fructosa), se observa la disminución de estas, posiblemente por avances en los procesos de fermentación natural.

Tabla 3. Azúcares presentes en el jugo de piña de rechazo de la variedad utilizada como materia prima

\begin{tabular}{cc} 
para la obtención de etano \\
\cline { 2 - 2 } Azúcar & $\mathbf{( \mathbf { g L } ^ { - 1 } )}$ \\
\hline Sacarosa & 2,94 \\
Glucosa & 75,62 \\
Fructosa & 80,27 \\
\hline Totales & 158,83 \\
\hline
\end{tabular}

Por otro lado, los resultados de las fermentaciones utilizando la levadura comercial $S$. cerevisiae y el microorganismo nativo de la misma variedad de piña se presentan en la figura $1 a$ y la figura $1 b$, respectivamente.

Adicionalmente, la figura 2 presenta la concentración de etanol en el tiempo para un experimento tomado como ejemplo, en este caso, la fermentación con $S$. cerevisiae en jugo de piña diluido $(75 \% \mathrm{v})$ con y sin adición de nutrientes para 24, 48 y 72 h de reacción.

Finalmente, la figura 3 muestra el perfil de la concentración de los azúcares y etanol para un sistema de fermentación que incluye la levadura S. cerevisiae y jugo de piña sin diluir (100 \%) en un medio sin suplementos o nutrientes adicionales, con temperatura y $\mathrm{pH}$ controlados $\left(30^{\circ} \mathrm{C}\right.$ y 4,5 , respectivamente). 
a)

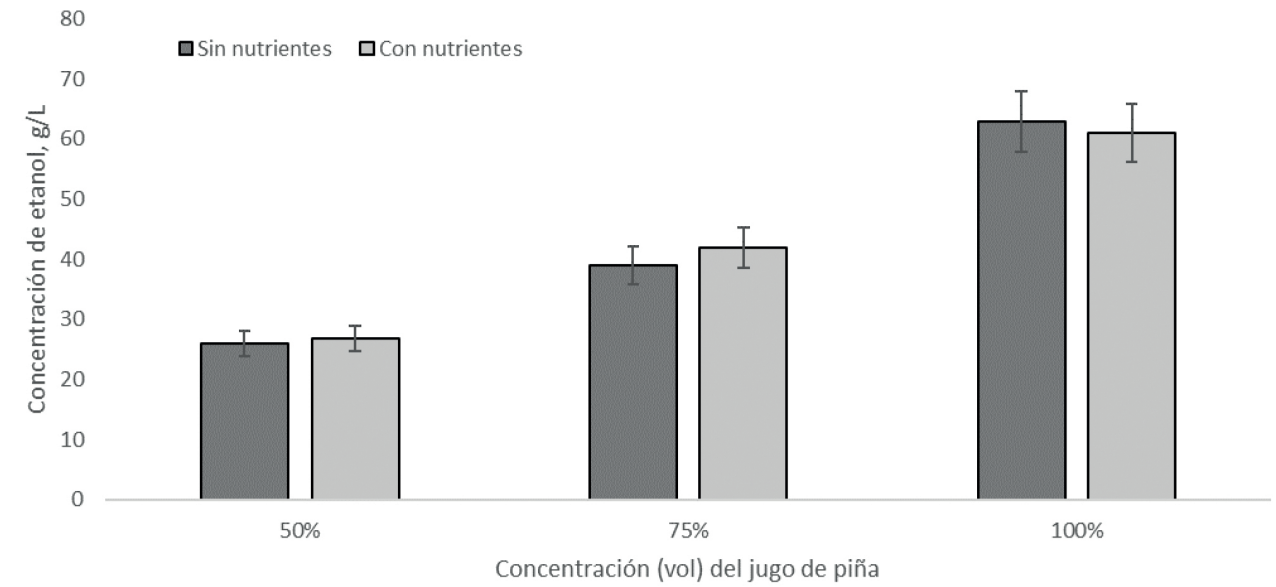

b)

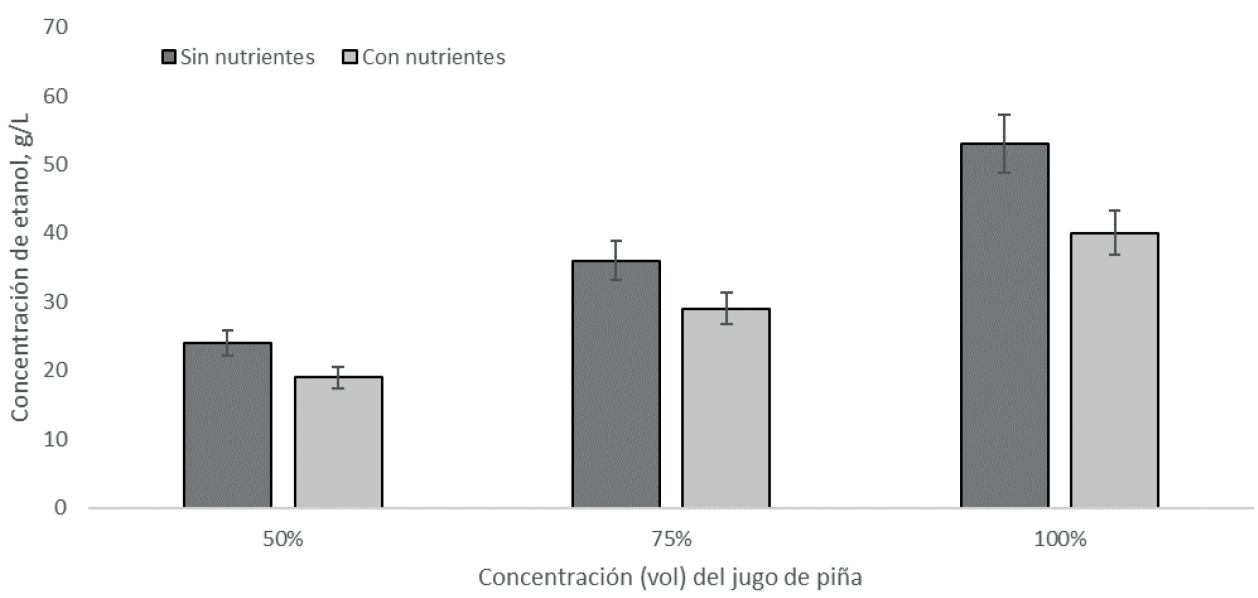

Figura 1. Producción de etanol a partir de jugo de piñas de rechazo utilizando diferente concentración de jugo de piña en los medios de cultivo, a) fermentación con S. cerevisiae, b) fermentación con microorganismos nativos de la piña.

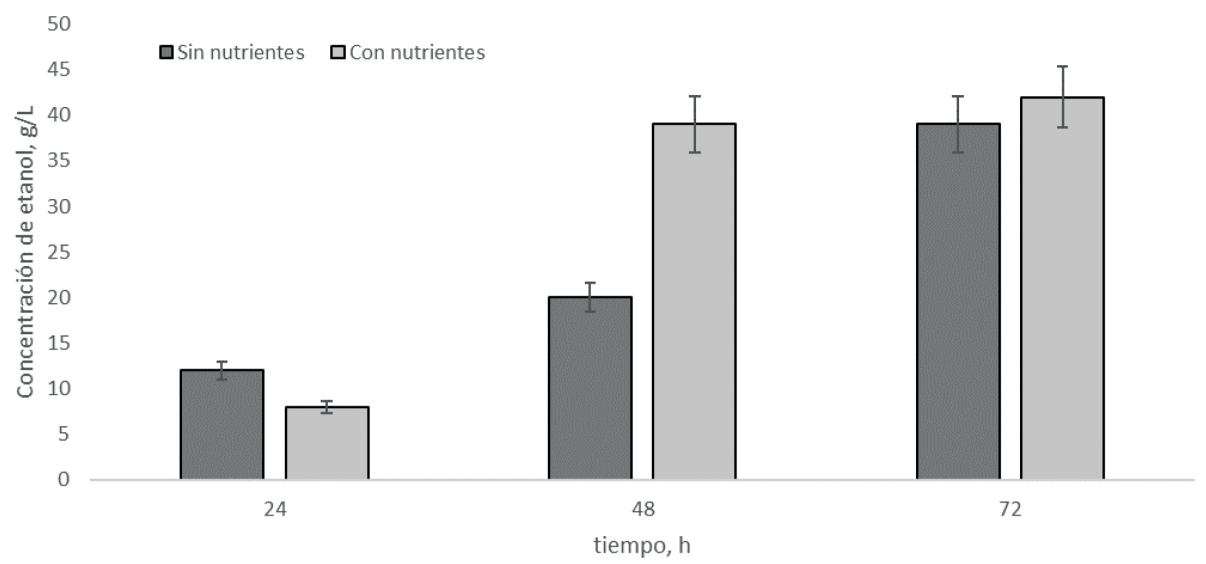

Figura 2. Producción de etanol a partir de jugo de piñas de rechazo para diferentes tiempos utilizando jugo diluido $(75 \%$ v) y levadura S. cerevisiae. 


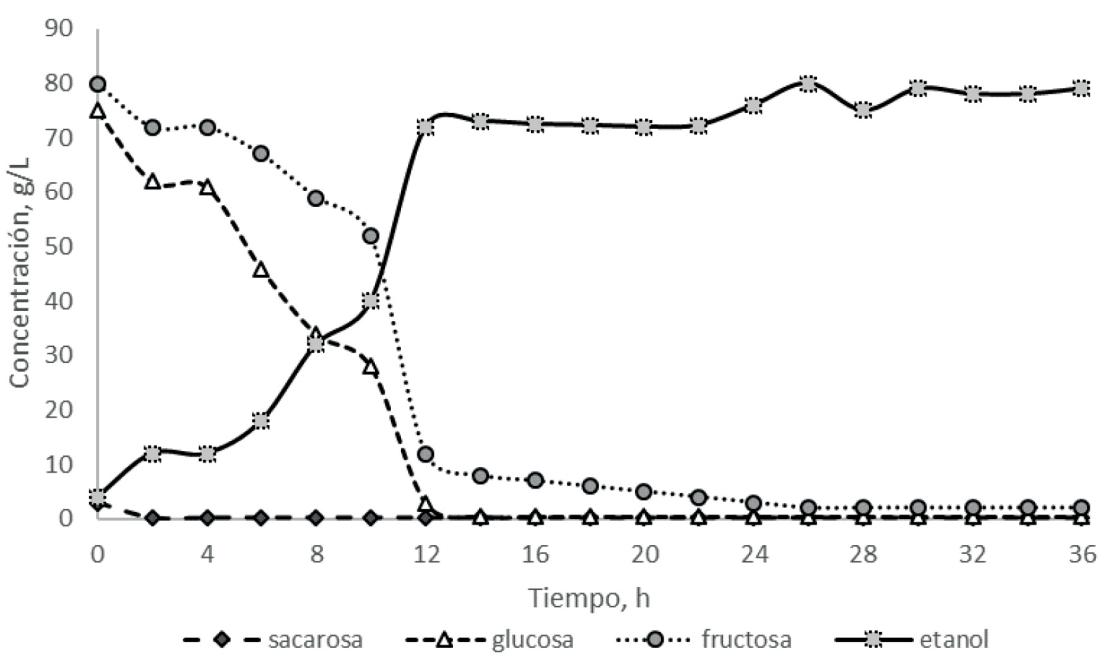

Figura 3. Perfil de concentración de azúcares y etanol para 36 h de fermentación con S. cerevisiae, jugo sin diluir, sin adición de nutrientes, $30{ }^{\circ} \mathrm{C}, \mathrm{pH}=4,5$.

\section{Discusión}

De acuerdo al análisis bromatológico de la tabla 2, la presencia de carbohidratos, lípidos (grasa), nitrógeno (proteína) y posibles trazas de metales (cenizas) proporciona elementos necesarios para el crecimiento de la levadura bajo condiciones de fermentación anaerobia. Al comparar la variedad de piña del Chocó con datos bromatológicos reportados para otras variedades de piña (como la Champaka y la Smoothe Cayenne), según [19], se observa que, en general, la piña del Chocó posee mayor proporción de carbohidratos y cenizas, y menor proporción de humedad y fibra cruda.

Con relación al perfil de azúcares (tabla 3), aparentemente, los microorganismos responsables de la degradación de los azúcares son selectivos al metabolizar la glucosa frente a la fructosa, pues se observa una mayor disminución de esta en la fruta de rechazo, comparada con la fruta fresca. Observaciones similares se encuentran reportadas en [20].

En cuanto al resultado de las fermentaciones, en las figuras 1 a y $1 \mathrm{~b}$ se observa que independientemente del microorganismo utilizado ( $S$. cerevisiae o nativo) e independiente y de la adición (o no) de nutrientes al medio, la dilución del jugo de piña afecta negativamente la producción de etanol, lo cual resulta razonable dada la menor concentración de azúcares fermentables por efectos de la dilución. En los experimentos donde se utiliza jugo de piña diluido al $50 \%$, la concentración de etanol a las $72 \mathrm{~h}$ resulta aproximadamente la mitad de la concentración de etanol, en el mismo tiempo, para aquellos experimentos que utilizan jugo de piña sin diluir $(100 \%)$. De los resultados se concluye que, para la obtención de etanol a partir de jugo de piñas de rechazo del departamento de Chocó, es conveniente utilizar el jugo sin diluir.

En cuanto a la adición de nutrientes, en la figura 1a se observa que para las fermentaciones con la levadura comercial no hay diferencias significativas en la producción de etanol cuando se adicionan o no suplementos al medio de reacción, independiente de la dilución del jugo. Aparentemente, para las condiciones experimentales utilizadas en este estudio (y para las 72 horas de fermentación), el medio natural ( $\sin$ nutrientes adicionales) proporciona los elementos necesarios y suficientes para el proceso de fermentación, por lo que la adición de nutrientes no es requerida. No obstante, de acuerdo a los resultados de la figura 2 , se puede inferir que el efecto de la adición de nutrientes se acentúa en la cinética del proceso, pues para el experimento mostrado como ejemplo en esa figura (jugo de piña $75 \% \mathrm{v}$ ), con la adición de nutrientes se alcanza una producción de etanol que alcanza aproximadamente los $40 \mathrm{gL}^{-1}$ desde las $48 \mathrm{~h}$ de proceso, sin cambios significativos para las 72 $\mathrm{h}$ de fermentación, mientras que sin la adición de nutrientes, aunque se alcanzan rendimientos similares para las $72 \mathrm{~h}$ de fermentación (crecano a los $40 \mathrm{gL}^{-1}$ ), a las $48 \mathrm{~h}$ el rendimiento solo alcanza aproximadamente el $50 \%$ de ese valor.

Por su parte, cuando se utiliza el microorganismo nativo (figura $1 \mathrm{~b}$ ), para las diferentes diluciones 
de jugo utilizadas y para las 72 h de fermentación, aparentemente, el uso de suplementos afecta de manera negativa la producción de etanol, pues se observan concentraciones de etanol inferiores en casi un $20 \%$ en los experiementos en que se adicionaron nutrientes en relación con aquellos que no los incluyeron. Dentro del alcance de esta investigación no es posible explicar el fenómeno, pero posiblemente se dé por efectos de eventual envenenamiento o desactivación de los microorganismos nativos en presencia de la cantidad o naturaleza de los suplementos utilizados como nutrientes, o bien porque estos microorganismos nativos requieran un mayor tiempo de activación, que lleva a un proceso de fermentación más lento, en comparación con el proceso donde se utiliza la levadura comercial.

Finalmente, al comparar las figuras $1 \mathrm{a}$ y $1 \mathrm{~b}$ se aprecia que, para las $72 \mathrm{~h}$ de reacción, hay mayor producción de etanol cuando se utiliza la levadura comercial que cuando se usa el microorganismo nativo. Al comparar entre sí los experimentos sin adición de nutrientes, en los medios diluidos se obtiene alrededor del $8 \%$ menos de etanol, y en el medio sin diluir, cerca del $16 \%$ menos de etanol cuando se usa el microorganismo nativo que cuando se usa la levadura comercial. Por su parte, cuando se adicionan nutrientes, la concentración de etanol que se obtiene para $72 \mathrm{~h}$ con el microorganismo nativo es casi un $30 \%$ inferior que la que se obtiene con la levadura S. cerevisiae, indicando nuevamente que la combinación de nutrientes añadidos con los microorganismos nativos no resulta favorable para la producción de etanol.

Dado que los experimentos mostrados en las figuras $1 \mathrm{a}$ y $1 \mathrm{~b}$ se realizaron sin control de temperatura, es posible que en el sistema se presenten fenómenos relacionados con el efecto de choques térmicos durante las diferentes etapas del proceso biotecnológico, puesto que la levadura no puede regular su temperatura y los procesos de fermentación alcohólica son exotérmicos, así que, cuando el calor generado no se retira, puede presentarse la desnaturalización de las proteínas y de los ácidos nucleicos de las células [21]. Por esa razón, los mejores resultados, es decir, los relacionados con el uso de levadura comercial en jugo sin diluir y sin adición de nutrientes, se replicaron en un sistema bajo temperatura y $\mathrm{pH}$ controlado (figura 3).
En la figura 3 se aprecia que la sacarosa se agota de forma inmediata, lo cual puede darse por su hidrólisis a glucosa y fructosa. En la figura 3 también puede observarse que la levadura comercial resulta selectiva a la glucosa, consumiéndola a una velocidad mayor en comparación a la fructosa. Por ejemplo, en aproximadamente $10 \mathrm{~h}$ de fermentación se ha consumido casi el $70 \%$ de la glucosa inicial, mientras que, para el mismo tiempo, de la fructosa inicial solo se ha consumido alrededor del $30 \%$. De otro lado, una vez se agota la glucosa (alrededor de $12 \mathrm{~h}$ ), la levadura aumenta la velocidad de consumo de fructosa. Este comportamiento permite inferir que estas se asimilan de forma simultánea, con preferencia sobre la glucosa, tal y como se reporta en [20], donde se atribuye el fenómeno a una multiplicidad de sistemas de captación de hexosas sobre la $S$. cerevisiae. Después de $13 \mathrm{~h}$ de fermentación, la glucosa residual es cercana a $0,43 \mathrm{gL}^{-1}$, la fructosa a $12 \mathrm{gL}^{-1}$ y la sacarosa es menor a $0,30 \mathrm{gL}^{-1}$, valores que, salvo el de la fructosa, que continúa disminuyendo a una velocidad relativamente lenta, se mantienen prácticamente constantes hasta casi las $40 \mathrm{~h}$ que se mantuvo la fermentación.

En cuanto al perfil de formación de etanol, se observa un contenido de etanol aproximado de 2,6 $\mathrm{gL}^{-1}$ al inicio de la fermentación (tiempo 0 en la figura 3). Este alcohol inicial puede producirse debido a los avances en los procesos de fermentación natural que se presentan en las piñas de rechazo por efecto de la actividad microbiana presente. También se observa que para 3 horas de fermentación ya existe una concentración de etanol cercana a los $12,0 \mathrm{gL}^{-1}$, indicando que el periodo de adaptación de los microorganismos en el medio es rápido, lo cual puede atribuirse a la preparación del inóculo en el mismo jugo de piña. También se observa, por comparación entre las figuras 1 y 3 , que al controlar la temperatura y el $\mathrm{pH}$ se aumenta significativamente la cinética de la reacción y se disminuye el tiempo requerido para el proceso de fermentación. De acuerdo con los resultados de la Figura 3, en un tiempo menor o igual a $13 \mathrm{~h}$ se agotan considerablemente los azúcares y la concentración de etanol permanece aproximadamente constante, en valores cercanos al valor máximo alcanzado a las $36 \mathrm{~h}$ (cercano a los $78 \mathrm{gL}^{-1}$ ), lo cual representa un significativo ahorro potencial en costos de producción. 
rev. ion. 2020;33(1):47-56. Bucaramanga (Colombia).

\section{Conclusiones}

Con base en los resultados de este trabajo, es posible concluir que el jugo obtenido a partir de las piñas de rechazo provenientes del corregimiento de Tutunendo en el departamento del Chocó representa una materia prima apta para la obtención de etanol a través de procesos de fermentación controlada con levadura comercial y sin necesidad de suplementos añadidos al medio. Esta materia prima contiene los carbohidratos y los nutrientes necesarios para alcanzar una producción de alcohol similar a la reportada en la literatura para otros sistemas de reacción o con diferentes materias primas. Adicionalmente, por tratarse de un desecho, puede considerarse una materia prima de bajo costo y su utilización para la obtención de productos de valor agregado (etanol) representa una potencial alternativa de desarrollo para la región y una disminución en el impacto ambiental negativo que su inadecuada disposición puede generar.

Así mismo, los resultados demuestran que el sistema utilizado es susceptible de ser mejorado $u$ optimizado en búsqueda de mejores condiciones de reacción que permitan incrementar los rendimientos o disminuir los tiempos del proceso. Por tanto, esto representa nuevos retos en investigación aplicada para buscar solución a problemáticas locales en el contexto nacional. Se espera, por lo tanto, que estos resultados permitan proponer y desarrollar alternativas para la valorización de los residuos del cultivo de piña de la región y para la disminución del impacto ambiental negativo que representan.

\section{Referencias bibliográficas}

[1] Ministerio de Agricultura y Desarrollo Rural. Balance del sector hortofrutícola en 2017. Bogotá, Colombia; 2017.

[2] González X. Panorama de la producción de piña en Colombia (sitio en internet). La República. Disponible en: https://www. agronegocios.co/agricultura/la-produccion-depina-en-colombia-llegaria-a-118-millones-detoneladas-al-finalizar-el-ano-2895397. Acceso en Mayo de 2019.

[3] Palacios JFM. Ensayos para la producción de etanol utilizando Ananas comosus de rechazo (tesis de grado). Medellín, Colombia: Universidad Pontificia Bolivariana; 2013.

[4] Palacios JFM, Barrera R, Zondervan E.
Process design and techno-economic analysis of a pineapple wine production plant under the context of the Choco-Colombia region. Comput. Aided Chem.Eng. 2019;46:277-82.

[5] Banerjee S, Ranganathan V, Pattic A, Arora A. Valorisation of pineapple wastes for food and therapeutic applications. Trends Food Sci. Tech. 2018;82:60-70.

[6] Gil L, Maupoey P. An integrated approach for pineapple waste valorisation. Bioethanol production and bromelain extraction from pineapple residues. J. Clean. Prod. 2018;172:1224-31.

[7] Sanewski G, Bartholomew D, Paull R. The pineapple: botany, production and uses. England: CAB International; 2018.

[8] Roda A, Lambri M. Food uses of pineapple waste and by-products: a review. Int. J. Food Sci. Technol. 2019;54:1009-17.

[9] Idiata D, Lyasele J. Waste To Wealth: Production of Bioethanol From Pineapple Waste. JMEST. 2014;1(4):282-7.

[10] Tropea A, Wilson D, La Torre L, Lo Curto R, Saugman P, Troy-Davies $P$, et al. Bioethanol production from pineapple wastes. J. Food Res. 2014;3(4):60-70.

[11] Santos A. Boletín informativo: Biocombustibles hoy No. 189 (sitio en internet). FedeBiocombustibles. Disponible en: https:// www.fedebiocombustibles.com/nota-webid-3064.htm. Acceso Mayo de 2019.

[12]Bullock GE. Review of emerging ethanol technologies. Bioenergy Rev. 2003;2(2):30-6.

[13] Lin Y, Tanaka S. Ethanol fermentation from biomass resources: current state and prospects. Appl. Microbiol Biotechnol. 2006;69:627-42.

[14]Alain K, Georges N, Aka Y. Ethanol production from pineapple cannery juice in Côte d'Ivoire with preselected yeast strains. J. Ferment Technol. 1987;65:475-581.

[15] Nigam J. Continuous ethanol production from pineapple cannery waste using immobilized yeast cells. J. Biotechnol. 2000;80(2):189-93.

[16] Rivera E, Costa A, Atala D, Maugeri F, Maciel M, Maciel R. Evaluation of optimization techniques for parameter estimation: Application to etanol fermentation considering the effect of temperature. Proc. Biochem. 2006;41:1682-7.

[17]Delgado-Huertas $H$, Arango-Wiesner $L$. Caracterización morfoagronómica de genotipos de piña (Ananas spp.) en un suelo 
de terraza alta de Villavicencio. Orinoquia. 2015;19(2):153-65.

[18]Cheftel J, Cheftel H. Introducción a la Bioquímica y Tecnología de los Alimentos (vol I). España: Acribia; 1992.

[19] United States Department of Agriculture. Basic report: 168193, Pineapple, raw, traditional varieties. Washington, United States; 2019.
[20]Kruckenberg A. The hexose transporter family of $S$. cereviciae. Arch. Microbiol. 1996;166(5):283-92.

[21] Ingledew WM. Alcohol production by $S$. cerevisiae: a yeast primer. In: The Alcohol Textbook. Jacques KA, Lyons TP, Kelsall DR, Editors. England: Nottingham University Press; 1999. p. 49-87. 